\title{
CT OF MULLERIAN ADENOSARCOMA ARISING IN ENDOMETRIOSIS
}

\author{
DAVID C. MCKAY, MD, JAMES H. ELLIS, MD, \\ AND CHARLES S. MARN, MD
}

Mullerian adenosarcoma is a rare tumor that usually arises in the uterus. Occasional cases arising in endometrial implants have been reported. We report the computed tomography (CT) findings of histologically proven mullerian adenosarcoma arising in perirectal endometriosis in a 46-year-old woman.

KEY WORDS:

Mullerian adenosarcoma; Endometriosis; Neoplasms; Computed tomography; Pelvic tumors

\section{INTRODUCTION}

Mullerian adenosarcoma (MA) is an uncommon variant of mullerian mixed tumor of the uterus generally characterized by a benign glandular component and a low grade sarcomatous stromal component (1). These tumors arise in endometrial tissue and thus can arise in ectopic foci of endometriosis. Cases of extrauterine MA arising from endometriosis of the ovary and of the pelvic wall have been described in the pathology literature (2-4). We present the computed tomography (CT) findings of a histologically proven case of $\mathrm{MA}$ arising in perirectal endometriosis.

\section{CASE REPORT}

A 46-year-old woman presented with a complaint of bright red blood per rectum and pelvic pain that increased with defecation. Her medical history was significant for endometriosis and pelvic pain dating back

From the Department of Radiology, University of Michigan Medical Center, Ann Arbor, Michigan.

Address reprint requests to: J.H. Ellis, MD, Department of Radiology, University of Michigan Medical Center, 1500 E. Medical Center Dr., Ann Arbor, MI 48109-0030.

Received August 1992; accepted September 1992.

Q 1993 Elsevier Science Publishing Co., Inc.

655 Avenue of the Americas, New York, NY 10010

$0899-7071 / 93 / \$ 6.00$ to 1965 . She had a hysterectomy for cervical dysplasia in 1975. No malignancy was found in the specimen. At laparotomy in 1982, fulguration of endometrial implants on the bladder and stomach and a bilateral salpingo-oophorectomy were performed. Numerous additional bowel implants were noted at the time of surgery but were not treated due to the length of the procedure. In 1985, she had a right hemicolectomy for endometriosis. She had a cholecystectomy in 1987 for endometriosis involving the gall bladder. In November 1990, she noted the onset of bright red rectal bleeding, and a biopsy taken during colonoscopy showed MA. She received a two-month course of iododeoxyuridine (IUdR) radiosensitization and radiation therapy from December 1990 to February 1991. Pre- and posttherapy CT scans (Figure 1) show the MA compressing the rectum and almost completely occluding its lumen. In March 1991 she underwent surgery to resect the sarcoma. A $10 \mathrm{~cm} \times 10 \mathrm{~cm}$ mass was resected, and another $15 \mathrm{~cm} \times 10 \mathrm{~cm}$ pelvic mass attached to the pelvic wall was felt to be unresectable. The histologic diagnosis was a pleomorphic malignant neoplasm with associated endometriosis consistent with mullerian adenosarcoma.

\section{DISCUSSION}

MA is an uncommon variant of malignant mesodermal mixed tumor (MMMT) that was first described in 1974 (1). It most commonly arises in the endometrium, but it has also been reported to arise in the endocervix and the myometrium (1). Extrauterine cases have been reported arising from the ovary (epithelium or endometrial implants) (4), pelvic sidewall (mesothelium or endometrial implants) $(3,4)$, and the pelvic peritoneum comprising the broad ligament (2). Recently, a case of MA arising in endometrial implants within the bladder has been reported (5).

The average age of occurrence for intrauterine MA 


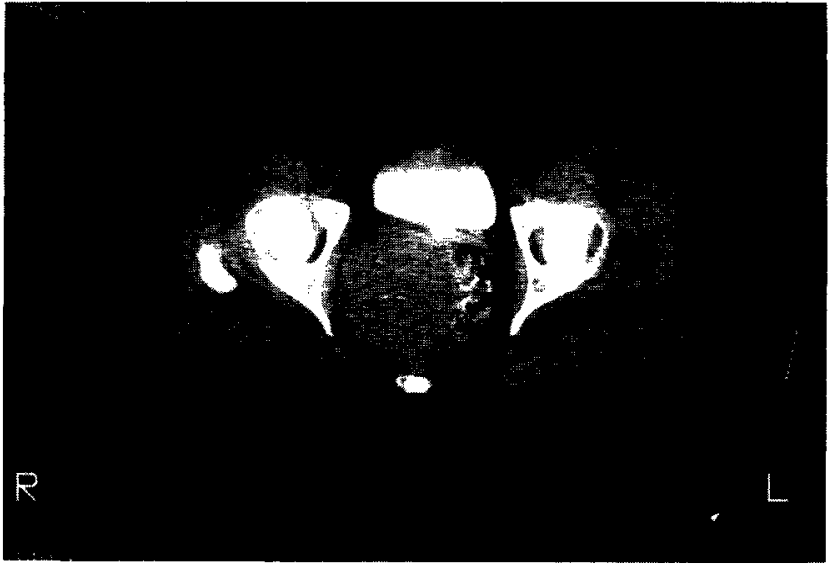

$\mathbf{A}$

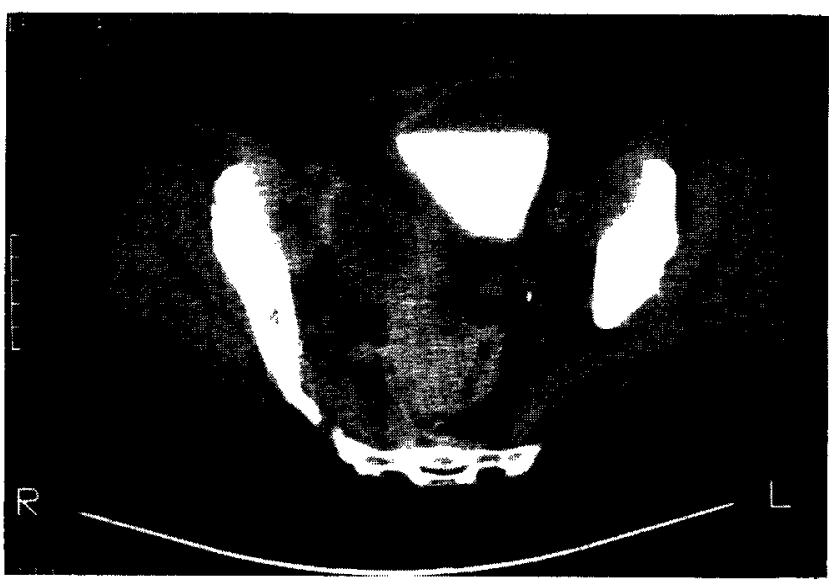

C

is 58 years and $60 \%$ of these patients are 50 years of age or older, although cases occurring as early as the second decade of life have been reported (1). Extrauterine MA has been reported to occur at a younger average age of 49 years (4).

Clinically, uterine MA often presents as abnormal vaginal bleeding, pelvic pain, or symptoms related to uterine prolapse. On physical exam, the most common findings are tissue protruding from the external cervical os or an enlarged uterus. Protruding tissue is often clinically and sometimes pathologically misinterpreted as benign endocervical or endometrial polyps (1). In the few reported cases of extrauterine MA, clinical presentation has been variable, depending in part on tumor location (2-5).

On gross pathology, intrauterine MA tumors are typically solitary masses confined to the uterus, usually as sessile or pedunculated polypoid or papillary masses within the endometrial cavity. They may contain small cysts, and are capable of becoming quite

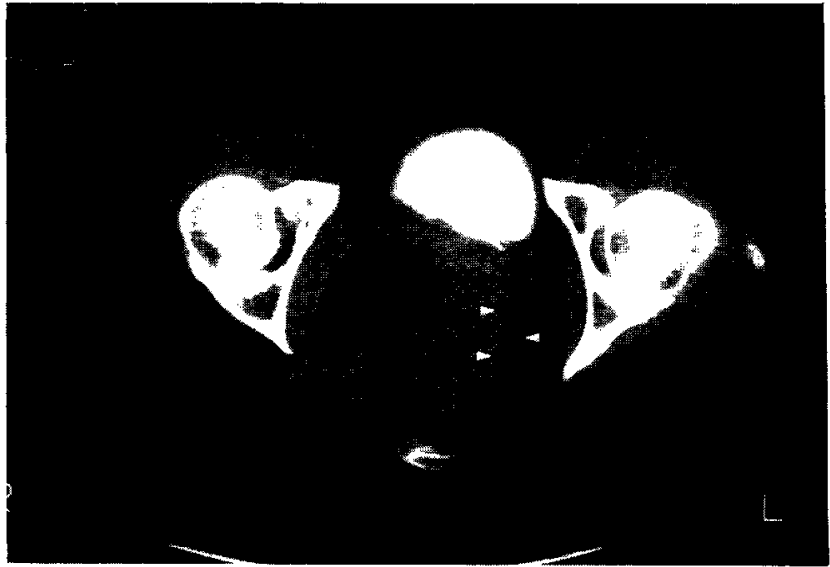

B

FIGURE 1. (A) Contrast-enhanced CT scan before radiation therapy shows a soft-tissue density, mostly homogeneous, well circumscribed lesion compressing the rectum and displacing the bladder anteriorly. (B) Post-therapy CT scan shows increased heterogeneity of the lcsion and continued compression of the rectum (arrowheads) with the lumen almost completely occluded. (C) Section from post-therapy CT scan obtained $5 \mathrm{~cm}$ superior to B shows low density, well circumscribed lesions consistent with endometriomas in the right pelvis, as wcll as additional solid tumor compressing bowel.

large, with tumors up to $17 \mathrm{~cm}$ greatest diameter having been reported (1). Extrauterine tumors are typically large, partly cystic masses that may have smooth or irregular and lobulated surfaces. They may displace or invade other pelvic organs (4). Intrauterine and extrauterine MA are identical at histologic examination, consisting of malignant sarcomatous stroma admixed with neoplastic, but histologically benign, epithelial elements. As a rule, these are tumors of low malignant potential, in contrast to the more typical MMMT in which the epithelial component is also malignant (1, 4). However, widespread hematogenous metastatic disease to lung, thoracic and lumbar vertebrae and mediastinal and abdominal perinodal adipose tissue have been reported, with only the sarcomatous element identified in the metastases $(3,4)$. There is some evidence that extrauterine MA is more aggressive than intrauterine MA (4).

Treatment of MA of the uterus is usually hysterectomy with bilateral salpingo-oophorectomy, although some cases have been treated with local excision, sometimes in combination with radiation therapy or chemotherapy (1). The disease recurs in 25 to $30 \%$ of patients, with recurrence almost always limited to the pelvis, vagina, or abdomen. A correlation between recurrence and deep myometrial invasion has been reported (6). The recommended treatment for recur- 
rent or primary extrauterine MA is resection, or wide local excision when complete removal is impossible. The role of chemotherapy in these cases is unknown.

Less is known about MA arising in foci of endometriosis, but it has been reported $(3,4)$. Ectopic endometrial tissue is subject to many of the changes that affect normal endometrium and it occasionally undergoes malignant change (7-9). It is difficult to assess the frequency with which this occurs, but it is clear that any type of malignant tumor that can arise from the uterine endometrium may also develop in ectopic endometrial tissue (10). The possibility exists that our case represents recurrent uterine tumor that was unrecognized in the hysterectomy specimen. However, in Clement's study (1), recurrence of uterine MA occurred at a mean interval of 3.4 years after hysterectomy and the longest interval reported was 9.5 years. As this patient's uterus was removed fifteen years before she was diagnosed with mullerian adenosarcoma, we believe that this is a primary tumor. In our opinion, this case meets all of Sampson's criteria (11) for malignant degeneration in endometriosis: (a) foci of endometriosis were found in close proximity to the tumor; (b) no other possible primary was found; and (c) the histological appearance is such that its origin from endometriosis is possible.

Although rare, MA should be considered in the differential diagnosis of a pelvic mass in a patient with a history of endometriosis. In our case, prior to therapy the tumor was solid in appearance, but this finding cannot separate this tumor from endometriosis alone, which also may present as a solid mass (12). Any evidence of metastasis in a patient with known or suspected endometriosis should raise the possibility of malignant degeneration.

\section{REFERENCES}

1. Clement PB, Scully RE. Mullerian adenosarcoma of the uterus: A clinicopathologic analysis of 100 cases with a review of the literature. Hum Pathol 1990;21:363-381.

2. Russell P, Slavutin L, Laverty R, Cooper-Booth J. Extrauterine mesodermal (mullerian) adenosarcoma: A case report. Pathology 1979;11:557-560.

3. Bard ES, Bard DS, Vargas-Cortes F. Extrauterine mullerian adenosarcoma: A clinicopathologic report of a case with distant metastases and review of the literature. Gynecol Oncol 1978;6:261-274.

4. Clement PB, Scully RE. Extrauterine mesodermal (mullerian) adenosarcoma: A clinicopathologic analysis of five cases. Am J Clin Pathol 1978;69:276-283.

5. Vara AR, Ruzics EP, Moussabeck O, Martin DC. Endometrioid adenosarcoma of the bladder arising from endometriosis. J Urol 1990;143:813-815.

6. Zaloudek CJ, Norris HJ. Adenofibroma and adenosarcoma of the uterus: A clinicopathologic study of 35 cases. Cancer 1981;48:354-366.

7. Brooks JJ, Wheeler JE. Malignancy arising in extragonadal endometriosis: A case report and summary of the world literature. Cancer 1977;40:3065-3073.

8. Brunson GL, Barclay DL, Sanders M, Araoz CA. Malignant extraovarian endometriosis: Two case reports and review of the literature. Gynecol Oncol 1988;30:123-130.

9. Shamsuddin A, Villa-Santa U, Tang CK, Mohamed NC. Adenocarcinoma arising from extragonadal endometriosis 14 years after total hysterectomy and bilateral salpingo-oophorectomy for endometriosis: Report of a case with ultrastructural studies. Am J Obstet Gynecol 1979;133:585-586.

10. Moustoufizadeh M, Scully RE. Malignant tumors arising in endometriosis. Clin Obstet Gynecol 1980;23:951-963.

11. Sampson JA. Endometrial carcinoma of the ovary arising in endometrial tissue of that tissue in that organ. Arch Surg $1925 ; 10: 1-72$

12. Fishman EK, Scatarige JC, Saksouk FA, Rosenshein NB, Siegelman SS. Computed tomography of endometriosis. J Comput Assist Tomogr 1983;7:257-264. 